\title{
Role of the excitation energy of the compound nucleus in binary decay processes
}

\author{
H. Paşca ${ }^{1,2, \star}$, A.V. Andreev ${ }^{1}$, G.G. Adamian ${ }^{1}$, and N.V. Antonenko ${ }^{1}$ \\ ${ }^{1}$ Joint Institute for Nuclear Research, 141980 Dubna, Russian Federation \\ 2 “Babeş-Bolyai" University, Faculty of Physics, 400084 Cluj-Napoca, Romania
}

\begin{abstract}
Employing the improved scission-point model, the isotopic and excitation energy trends of the charge distribution of fission fragments are studied in fission of eveneven Th isotopes at low and high excitation energies.
\end{abstract}

\section{Introduction}

As known, with increasing neutron number of fissioning even-even Th isotopes the transition from one-peaked to two-peaked charge distribution of fission fragments occurs through transient threepeaked shape in which the symmetric and asymmetric components of the distribution have almost equal weights [1]. The general opinion is that the competition between symmetric and asymmetric fission modes is related to the deformed shells. However, in the case of high-energy neutron-induced fission of ${ }^{238} U$ [2], the experimental data show that the mass distribution maintains two-peaked shape even at neutron energies of 50-60 MeV. At these excitation energies, the shell effects are expected to be washed out, and the nucleus is supposedly left with a dominant symmetric mode, that is contrary to the experiments. The experimental data on fission of ${ }^{180} \mathrm{Hg}$ [3] also suggest that the asymmetric mass distribution can not be anticipated only from the microscopic effects in the fragments. Our aim is to explain the transformation of the charge distribution with neutron number in the electro-magneticinduced fission $\left(E_{\gamma}=11 \mathrm{MeV}\right)$ of Th isotopes and to predict the charge distributions at large excitation energies. The fission is described within the improved scission-point model [4]. The most important ingredient of the model is the potential energy of system as a function of charge (mass) asymmetry, deformations of the fission fragments, and internuclear distance. The knowledge of the deformations of nascent fragments at scission point is also crucial.

\section{Statistical scission-point model}

The statistical scission-point model relies on the assumption that the statistical equilibrium is established at scission where the observable characteristics of fission process are formed. At the scission point the decaying system is modeled by two uniformly charged, nearly touching coaxial ellipsoids - the fission fragments forming the dinuclear system (DNS). By taking into consideration the volume conservation, the system is completely defined by the mass and charge numbers $\left(A_{i}, Z_{i}\right)$

^e-mail: pasca@theor.jinr.ru 
of the fragments, deformation parameters of fragments, $\beta_{i}$, and interfragment distance $R$. Here, the index $i$ designates the light or heavy fragment of the DNS $(i=L, H)$. The potential energy $U=B_{L}+B_{H}+V$ of the DNS consists of the binding energies $B_{i}$ of the fragments and the mutual fragment-fragment interaction potential $V$ [4]. The binding energy $B_{i}=U_{i}^{L D}+\delta U_{i}^{\text {shell }}$ of each fragment consists of the excitation-energy-dependent liquid-drop energy $U_{i}^{L D}$ and deformation-dependent shell-correction term $\delta U_{i}^{\text {shell }}$ calculated with the two-center shell model. The damping of the shell corrections with excitation energy is taken into account. The interaction potential $V=V^{N}+V^{C}$ consists of the Coulomb interaction potential $V^{C}$ of the two uniformly charged ellipsoids and nuclear interaction potential $V^{N}$ in the double-folding form [4]. The interaction potential presents an inner potential pocket and external barrier which prevents the system from decay. The barrier, $B_{q f}\left(A_{i}, Z_{i}, \beta_{i}\right)=V\left(A_{i}, Z_{i}, \beta_{i}, R_{b}\right)-V\left(A_{i}, Z_{i}, \beta_{i}, R_{m}\right)$, calculated as the difference of the interaction potentials at the top of the external barrier $\left[R=R_{b}\left(A_{i}, Z_{i}, \beta_{i}\right)\right]$ and at the bottom of the potential pocket $\left[R=R_{m}\left(A_{i}, Z_{i}, \beta_{i}\right)\right]$, prevents the decay of the DNS in $R$. The height $B_{q f}$ of barrier has also an impact on the yields. With increasing elongation and decreasing charge (mass) asymmetry the value of $B_{q f}$ decreases, the system becomes more unstable and decays. Because the dynamical treatment is not explicitly performed here, we simulate the dynamical effects by restricting the minimum value of the height $B_{q f}$ of the decay barrier. In the calculations, we take into consideration only those configurations for which $B_{q f}$ is larger than $\sim 1 \mathrm{MeV}$. This condition restricts the highly deformed unstable configurations in the $\left(\beta_{L}, \beta_{H}\right)$ plane.

Because the thermal equilibrium is assumed at the scission point, the excitation energy $E^{*}\left(A_{i}, Z_{i}, \beta_{i}, R_{m}\right)=E_{C N}^{*}+\left[U_{C N}(A, Z, \beta)-U\left(A_{i}, Z_{i}, \beta_{i}, R_{m}\right)\right]$ at scission is calculated as the initial excitation energy of the fissioning nucleus $E_{C N}^{*}$ plus the difference between the potential energies of fissioning compound nucleus $U_{C N}(A, Z, \beta)$ and of the system at the scission point $U\left(A_{i}, Z_{i}, \beta_{i}, R_{m}\right)$ [4]. Note that the value of $R_{m}$ is taken directly from the interaction potential and it is deformationdependent, which allows us to have a precise definition of the scission configuration and excitation energy. The relative formation probability of the DNS with particular masses, charges and deformations of the fragments is calculated as [4]:

$$
w\left(A_{i}, Z_{i}, \beta_{i}, E^{*}\right)=N_{0} \exp \left[-\frac{U\left(A_{i}, Z_{i}, \beta_{i}, R_{m}\right)+B_{q f}\left(A_{i}, Z_{i}, \beta_{i}\right)}{T}\right],
$$

where $N_{0}$ is the normalization factor. In Eq. (1), the temperature is calculated as $T=\sqrt{E^{*} / a}$, where $a=\left(A_{L}+A_{H}\right) / 12 \mathrm{MeV}^{-1}$ is the level density parameter, which seems to be a good approximation for the systems studied here [4]. Note that in the literature various values for $a$ are used (e.g. $a=$ $\left(A_{L}+A_{H}\right) / 12$ to $\left.a=\left(A_{L}+A_{H}\right) / 8\right)$. In our calculations, a single value of the temperatureis used at the global potential minimum of $U$ before the shell damping. Variations of the level density parameter in this work lead to minute changes in the results.

In order to obtain the mass-charge distribution $Y\left(A_{i}, Z_{i}, E^{*}\right)$ of fission fragments, one should integrate (1) over $\beta_{L}$ and $\beta_{H}$. For the calculations of mass and charge distributions, the following expressions are used:

$$
Y\left(A_{i}, E^{*}\right)=\frac{\sum_{Z_{i}} Y\left(A_{i}, Z_{i}, E^{*}\right)}{\sum_{Z_{i}, A_{i}} Y\left(A_{i}, Z_{i}, E^{*}\right)}, Y\left(Z_{i}, E^{*}\right)=\frac{\sum_{A_{i}} Y\left(A_{i}, Z_{i}, E^{*}\right)}{\sum_{Z_{i}, A_{i}} Y\left(A_{i}, Z_{i}, E^{*}\right)} .
$$

\section{Results and discussions}

In Fig. 1 the calculated mass distributions resulting from the spontaneous fission of even-even nuclei Cf and Fm with $A=250-256$ are depicted. The results seem to be in good agreement with the experimental data compiled and shown schematically in Fig. 8 of Ref. [5]. The calculated 

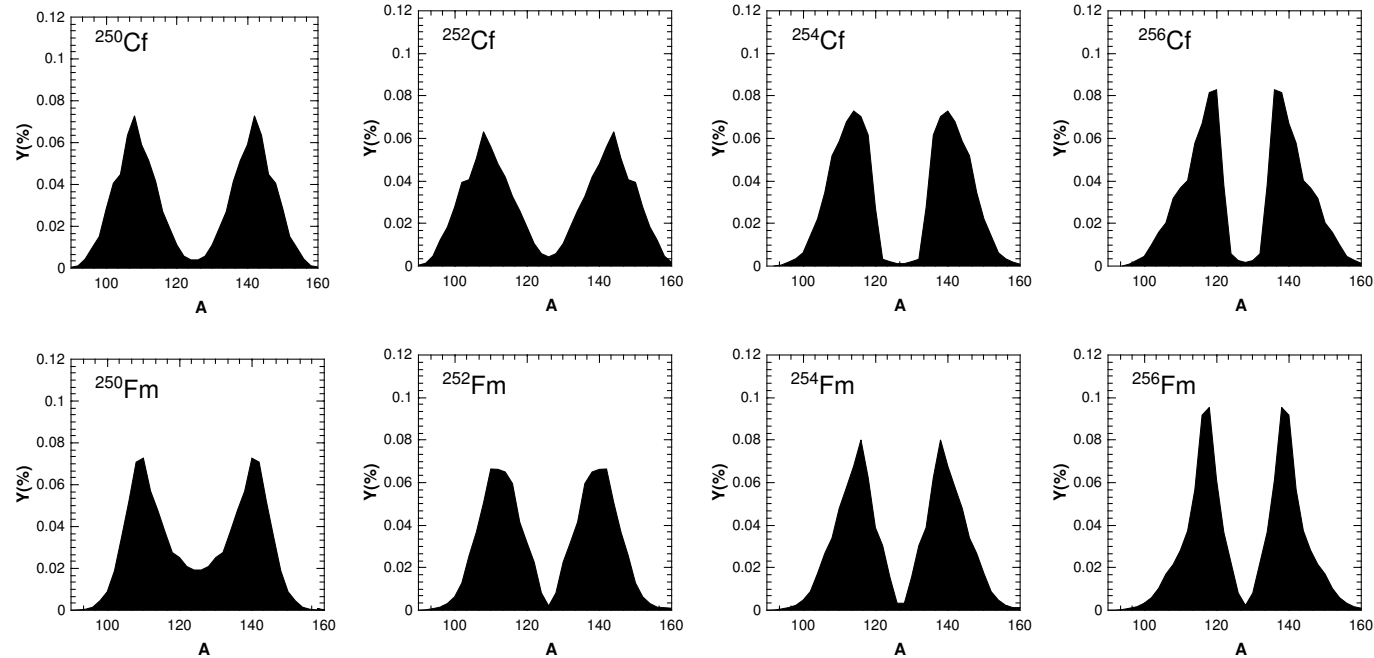

Figure 1. The calculated mass distributions in the spontaneous fission of the indicated even-even actinides.
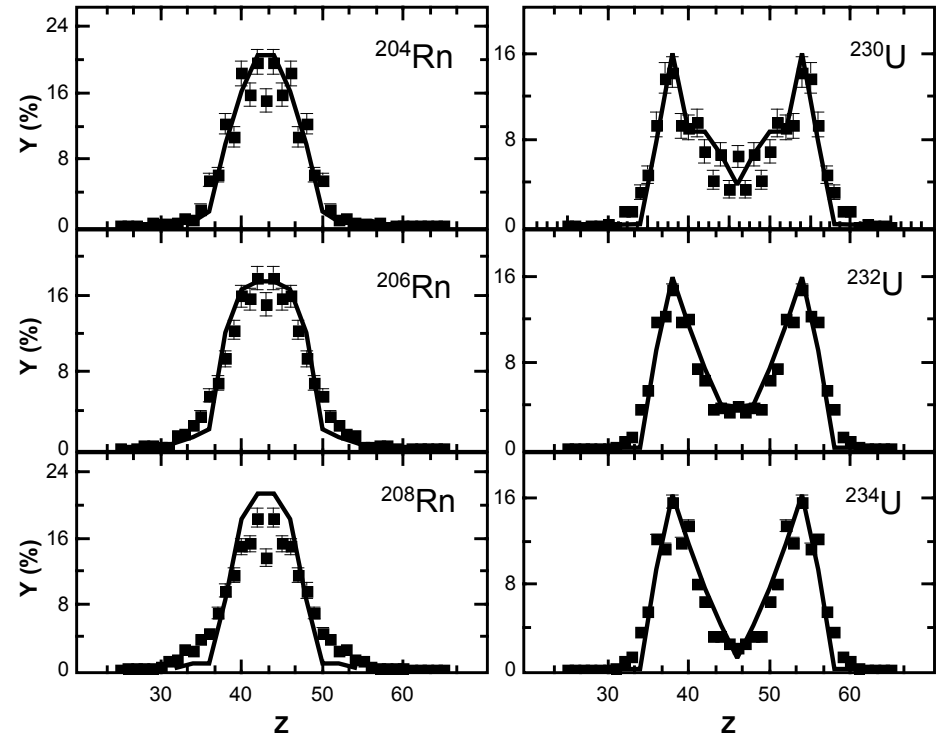

Figure 2. The calculated charge distributions (lines) for electro-magnetic-induced fission of the indicated radon and uranium isotopes at $11 \mathrm{MeV}$ excitation energy are compared with the experimental data [1] (symbols). The lines connect the calculated points for even-even fission fragments.

charge distributions resulting from the electro-magnetic-induced $\left(E_{\gamma}=11 \mathrm{MeV}\right)$ fission of eveneven ${ }^{204,206,208} \mathrm{Rn},{ }^{230,232,234} \mathrm{U}$, and ${ }^{222,224,226,228} \mathrm{Th}$ nuclei are compared with the experimental data of Ref. [1] in Figs. 2 and 3. For the isotopes of Rn (Fig. 2) and ${ }^{222}$ Th (Fig. 3), the fragment charge distributions are clearly symmetric, with a single prominent peak around $Z / 2$, while the charge distributions of fissioning nuclei ${ }^{230,232,234} \mathrm{U}$ (Fig. 2) and ${ }^{228} \mathrm{Th}$ (Fig. 3) have two distinct asymmetric peaks. The fission of the ${ }^{226} \mathrm{Th}$ nucleus shows a three-peaked distribution (Fig. 3). As seen, our model is suitable for describing both symmetric and asymmetric charge distributions. The transition from one-peaked to two-peaked distribution, going through transient three-peaked shape, can be explained 
by studying the driving potential:

$$
\left\langle U\left(Z_{i}\right)\right\rangle=\frac{\sum_{A_{i}} \int d \beta_{L} d \beta_{H} U\left(A_{i}, Z_{i}, \beta_{i}, R_{m}\right) w\left(A_{i}, Z_{i}, \beta_{i}, E^{*}\right)}{\sum_{A_{i}} \int d \beta_{L} d \beta_{H} w\left(A_{i}, Z_{i}, \beta_{i}, E^{*}\right)}
$$

averaged over the mass number $A_{i}$ and deformations $\beta_{L, H}$. In the case of ${ }^{222} \mathrm{Th}$ isotope the driving potential $\left\langle U\left(Z_{i}\right)\right\rangle$ shows a deep minimum at $Z / 2=45$. For the ${ }^{224} \mathrm{Th}$ nucleus, $\left\langle U\left(Z_{i}\right)\right\rangle$ starts displaying the formation of the second minimum around $\mathrm{Kr}-\mathrm{Sr}$, while for the ${ }^{226} \mathrm{Th}$ isotope, the second minimum becomes almost as deep as the central (symmetric) one. Even though that the values of $\left\langle U\left(Z_{L}=\right.\right.$ $36-38)\rangle$ is slightly higher than $\left\langle U\left(Z_{L}=44\right)\right\rangle$, the corresponding yields are the same. This is easily explained by the fact that in $\left(\beta_{L}, \beta_{H}\right)$ plane, the minima for $\mathrm{Kr}-\mathrm{Sr}$ are wider than those for $\mathrm{Ru}-$ $\mathrm{Pd}$ [4]. For the ${ }^{228} \mathrm{Th}$ nucleus, the asymmetric minima become deeper, facilitating the asymmetric fragmentations, but the central minimum still remains.

To understand the conditions under which the mass-asymmetric minimum appears in the driving potential, we analyze all components of the driving potential: the liquid-drop energy $\left\langle U^{L D}\left(Z_{i}\right)\right\rangle=$ $\left\langle U_{L}^{L D}\right\rangle+\left\langle U_{H}^{L D}\right\rangle$, the interaction potential $\left\langle V\left(Z_{i}\right)\right\rangle=\left\langle V^{C}\right\rangle+\left\langle V^{N}\right\rangle$, and the shell-correction energy $\left\langle\delta U^{\text {shell }}\left(Z_{i}\right)\right\rangle=\left\langle\delta U_{L}^{\text {shell }}\right\rangle+\left\langle\delta U_{H}^{\text {shell }}\right\rangle$ (Fig. 4).
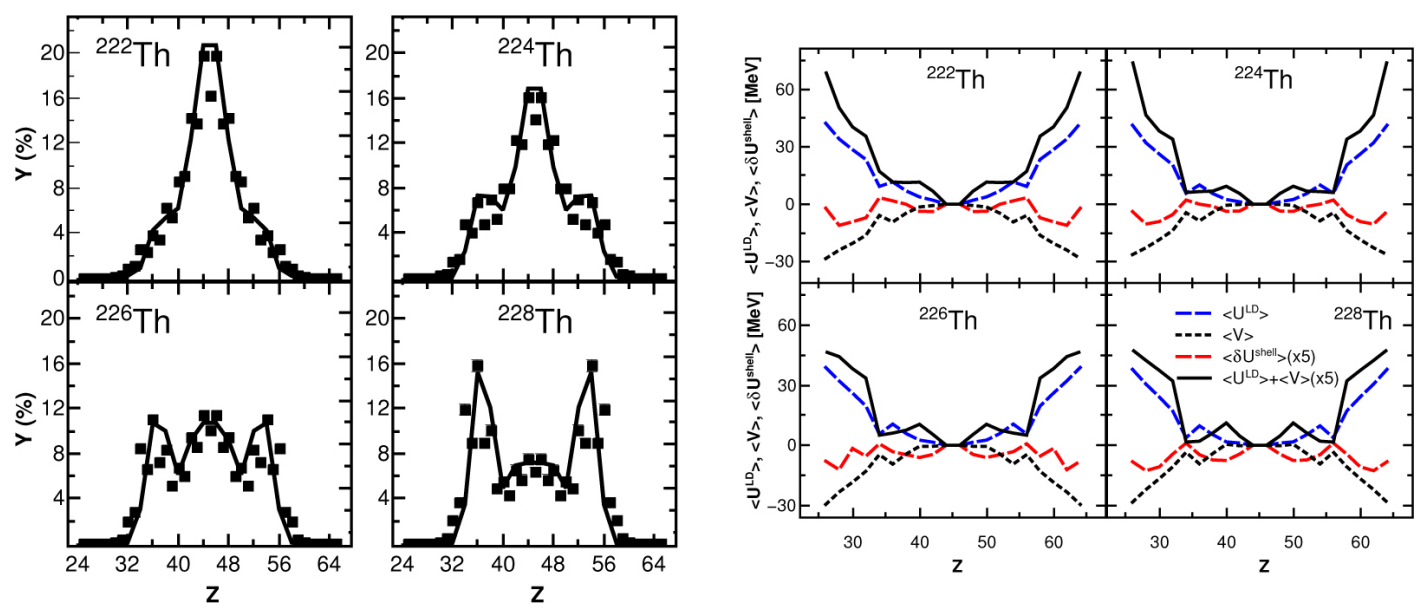

Figure 3. Left side: the same as in Fig. 2, but for Th isotopes. Right side: the calculated components of the driving potentials $\left\langle U\left(Z_{i}\right)\right\rangle$ at $11 \mathrm{MeV}$ (solid black lines) excitation energy of the initial compound nucleus for indicated fissioning thorium isotopes. The liquid-drop energy $\left\langle U^{L D}\right\rangle$ (dashed blue lines), the nucleus-nucleus interaction potential $\langle V\rangle$ (dotted lines), and the shell-correction energy $\left\langle\delta U^{\text {shell }}\right\rangle$ (dashed red lines) are normalized to the zero energy at $Z_{L}=44$. The values of $\left\langle\delta U^{\text {shell }}\right\rangle$ and $\left\langle U^{L D}\right\rangle+\langle V\rangle$ are scaled by a factor of 5 , for visibility.

The liquid-drop energy increases globally when the charge (mass) number deviates from $Z_{C N} / 2$ $\left(A_{C N} / 2\right)$. The interaction potential has the opposite global behavior. These two components depend on deformations of the DNS nuclei that create local minima in the $\left\langle U^{L D}\left(Z_{i}\right)\right\rangle$ and $\left\langle V\left(Z_{i}\right)\right\rangle$. The larger deformations result in the smaller interaction energy and the larger liquid-drop energy. The position of minimum in the $\left(\beta_{L}, \beta_{H}\right)$ plane depends on the shell corrections. For the nuclei with $Z_{L}=40-44$, the shell effects are relatively weak because $Z_{L}\left(N_{L}\right)$ is midway between proton (neutron) shell closures. This leads to an almost pure liquid drop behavior of the scission configuration and larger deformations of the fragments. Thus, the asymmetric minimum in the driving potential appears due to the interplay between the liquid-drop energy and the nucleus-nucleus interaction potential. The shell effects also 

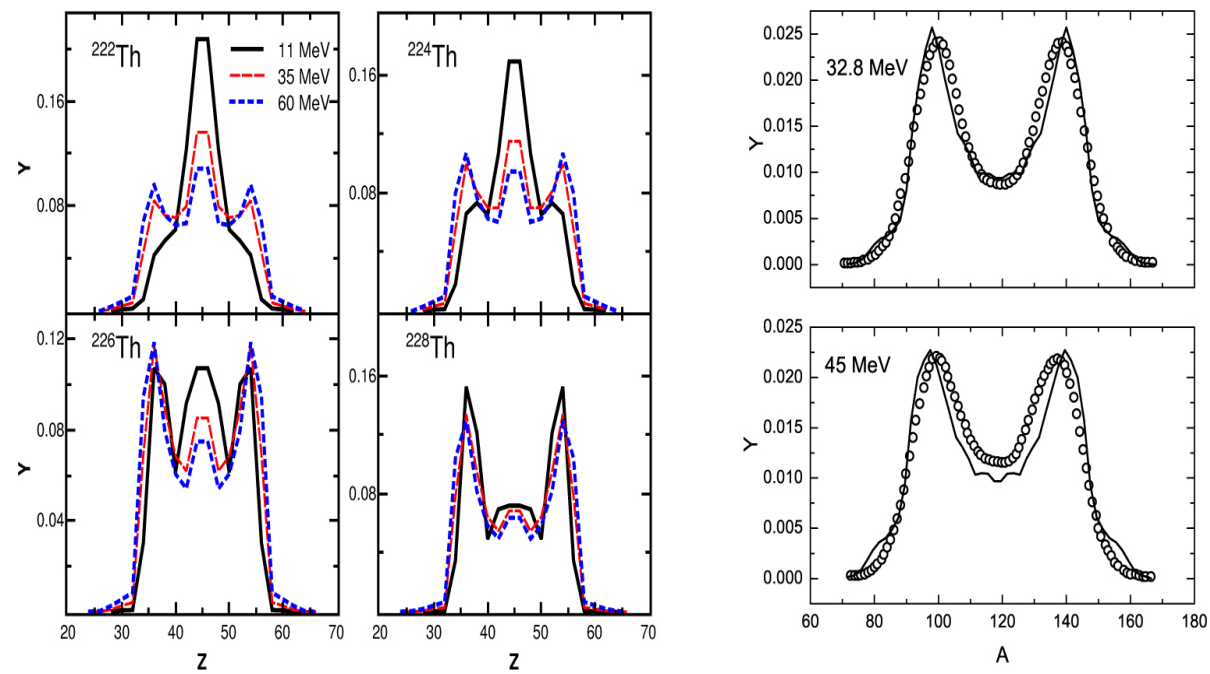

Figure 4. Left side: the calculated charge distributions for ${ }^{222,224,226,228} \mathrm{Th}$ isotopes at 11,35 , and $60 \mathrm{MeV}$ excitation energies of the initial compound nucleus. Right side: the calculated mass distribution resulting from the ${ }^{238} U(n, f)$ reaction at the indicated energies. The experimental data are from [2].

indirectly affect (through the deformations of nuclei) the appearance of the asymmetric minimum. The direct role of the shell effects is expressed by changing the position and depth of this minimum in the driving potential. For example, for ${ }^{226} \mathrm{Th}$, at $Z_{L}=34,36$, and 38 the DNS nuclei are near magic nuclei with $N_{L}=50$ and $N_{H}=82$. This implies high values of their stiffness parameter and so the minimum in the $\left(\beta_{L}, \beta_{H}\right)$ plane is situated at small deformations. As a result, at $Z_{L}=34-38$ the $\langle V\rangle$ decreases faster than the $\left\langle U^{L D}\right\rangle$ increases. At $Z_{L}<34$ the increase of the $\left\langle U^{L D}\right\rangle$ overcompensates the decrease of the $\langle V\rangle$, and, correspondingly, the asymmetric minimum appears at $Z_{L}=34$. The shell corrections cause the shift of the minimum from $Z_{L}=34$ to $Z_{L}=36$ and make this minimum deeper in the driving potential (Fig. 3). It is interesting to note that only in the cases of ${ }^{222,224} \mathrm{Th}$ the shell-correction energy $\left\langle\delta U^{\text {shell }}\left(Z_{i}\right)\right\rangle$ diminishes the asymmetric minimum of the liquid-drop energy plus the interaction potential in Fig. 3. With increasing $N-Z$ of the fissioning nucleus the liquid-drop energy/interaction potential increases slower/faster with charge asymmetry. This is the reason of the appearance of the pronounced asymmetric minimum in the cases of ${ }^{226,228} \mathrm{Th}$.

The predicted charge distributions of fissioning nuclei ${ }^{222,224,226,228} \mathrm{Th}$ at large excitation energies are given in the left part of Fig. 4. The calculated mass distributions of fissioning ${ }^{239} \mathrm{U}$ (lines) at two excitation energies (mentioned in the figure legends), describing the experimental data [2] (open circles) are plotted in the right part of Fig. 4. The distributions widens with increasing excitation energy for all nuclei. For ${ }^{222,224} \mathrm{Th}$ isotopes, the central peak recedes, and two asymmetric peaks appear suppressing the symmetric mode. The charge distributions of ${ }^{226,228} \mathrm{Th}$ at higher excitation energies (Fig. 4) emphasize the asymmetric aspect of the distribution, making the symmetric peak smaller and narrower, and the asymmetric maxima wider and taller. This is easily explained by the fact that with higher excitation energy the previously unaccessible asymmetric configurations are involved. The average driving potentials match the yields at 35 and $60 \mathrm{MeV}$ excitation energy. At $60 \mathrm{MeV}$ excitation energy $\left\langle U\left(Z_{i}\right)\right\rangle$ has asymmetric minima resulting in larger yields of asymmetric mode. The excitation energy reduces the shell effects that causes a widening of the minima in the deformation space $\left(\beta_{L}, \beta_{H}\right)$ and a migration of the minima in the potential-energy surface. In the case of fissioning $\mathrm{Th}$ isotopes for $\mathrm{Se}-\mathrm{Kr}$ charge fragmentations the minima widen with excitation energy 
faster than for the symmetric fragmentations. However, the influence of the excitation energy is not so dramatic. The deformations $\beta_{L}\left(Z_{L}\right)$ and $\beta_{H}\left(Z_{L}\right)$ of fragments, corresponding to the asymmetric minimum of the driving potential, do not change strongly, especially for the configurations with magic or close to magic nuclei $\mathrm{Se}-\operatorname{Kr}\left[N_{L}=50\right.$ and $\left.N_{H}=82\right]$. So, the deformations of the light nuclei with $Z_{L}=34-36$ remain to be smaller than the deformations of the DNS nuclei with $Z_{L}=38-44$. As a result, the $\left\langle V\left(Z_{i}\right)\right\rangle$ decreases faster than the $\left\langle U^{L D}\left(Z_{i}\right)\right\rangle$ increases from $Z_{L}=38$ to $Z_{L}=34$. At $Z_{L}<34$ their behaviors are opposite, and the minimum at $Z=34$ is produced. So, the asymmetric minimum in the driving potential is produced due to the deformation effect. With increasing excitation energy of the fissioning nucleus, the liquid-drop energy $\left\langle U^{L D}\left(Z_{i}\right)\right\rangle$ increases slower with charge asymmetry. This leads to the pronounced asymmetric minimum in the cases of ${ }^{222,224} \mathrm{Th}$.

\section{Conclusions}

In this paper it is shown that the improved scission-point model can be a suited tool for the description of the symmetric and asymmetric charge (mass) fragmentations resulting from the low-energy fission of different nuclei. The transition from a symmetric to an asymmetric fragmentation of Th nuclei is attributed to the interplay between the competing liquid-drop surface energy and the interaction energy in the driving potential of these systems at the scission point. This interplay depends on the excitation energy and the isospin of the fissioning nucleus. The shell effects affect indirectly through the deformations of nuclei on the appearance of the asymmetric minimum. The three-equal-peaked charge distribution is not the specific property of the ${ }^{226} \mathrm{Th}$ nucleus. The shape of the charge distribution depends on the excitation energy. For the fissioning nucleus ${ }^{226} \mathrm{Th}$ at high excitation energies, the charge distributions are well asymmetric but not the three-equal-peaked ones. The experimental verification of this unexpected effect is desirable.

\section{References}

[1] K.-H. Schmidt et al., Nucl. Phys. A 665, 221 (2000)

[2] I.V. Ryzhov et al., Phys. Rev. C 83, 054603 (2011)

[3] A.N. Andreyev, M. Huyse, P. Van Duppen, Rev. Mod. Phys. 85, 1541 (2013)

[4] H. Paşca, A.V. Andreev, G.G. Adamian, N.V. Antonenko, Phys. Rev. C 94, 064614 (2016); Phys. Lett. B 760, 800 (2016); Eur. Phys. J. A 52, 369 (2016)

[5] S. Hofmann and G. Münzenberg, Rev. Mod. Phys. 72 , 733 (2000) 